\title{
Video-assisted thoracoscopic thymectomy for myasthenia gravis: where we are
}

\author{
Tugba Cosgun ${ }^{1}$, Alper Toker ${ }^{2}$
}

${ }^{1}$ Department of Thoracic Surgery, Istanbul Bilim University, Istanbul, Istanbul; ${ }^{2}$ Department of Thoracic Surgery, Istanbul Florence Nightingale Hospital, Istanbul, Turkey

Contributions: (I) Conception and design: All authors; (II) Administrative support: All authors; (III) Provision of study materials or patients: A Toker; (IV) Collection and assembly of data: T Cosgun; (V) Data analysis and interpretation: T Cosgun; (VI) Manuscript writing: All authors; (VII) Final approval of manuscript: All authors.

Correspondence to: Alper Toker. Department of Thoracic Surgery, Istanbul Florence Nightingale Hospital, Istanbul, Turkey. Email: salpertoker@gmail. com; alper.toker@florence.com.tr.

\begin{abstract}
Thymectomy has long been considered one of the treatment modalities for myasthenia gravis (MG). The centre of debate has been the surgical technique for thymectomy, such as complete, extended and maximal thymectomies, and the surgical approach with the transsternal approach being the most common. Partial sternal splitting was the gold standard approach for many decades with less postoperative complications and safety. Transcervical thymectomy and video-assisted thoracoscopic surgery (VATS) thymectomy gained popularity for superior cosmesis, less invasive approach, shorter hospital stays and increased safety. Both techniques may provide a complete removal of the thymus gland. Now, different VATS techniques are preferred by different surgeons and clinics. The debate is shifting to the options of an extended thymectomy or a bigger thymus resection with minimally invasive techniques. Robotic thymectomy, which is considered a robot-assisted VATS thymectomy, became an important choice for surgical treatment in several centres. In this study, we aimed to study the role of thymectomy in the treatment of MG, including debatable indications and recently developed attitudes for the difficult decisions, and the effects of recent technology on the outcome of MG treatment.
\end{abstract}

Keywords: Myasthenia gravis (MG); video-assisted thoracoscopic surgery (VATS); robot-assisted thoracoscopic surgery

Received: 10 April 2018; Accepted: 15 May 2018; Published: 09 August 2018.

doi: $10.21037 /$ jovs.2018.07.11

View this article at: http://dx.doi.org/10.21037/jovs.2018.07.11

\section{Introduction}

German surgeon Sauerbruch performed the first thymectomy more than 100 years ago. In 1903, in Breslau, a low-pressure chamber for operating on the open thorax was developed by Sauerbruch. In this way, he introduced advances in thoracic surgery. He developed the transcervical approach for thymectomy in 1911, in Zurich, Switzerland (1). Although the first thymectomy was performed by Sauerbruch, Alfred Blalock described the anatomical basis for the surgical technique 20 years later. In 1936, Blalock performed a transsternal complete thymectomy on a patient for a thymoma associated with severe MG. The patient's MG markedly improved for several years. After this successful case, Blalock and his associates reported treating patients with MG by thymectomy (2). In 1939, he demonstrated a positive clinical response in patients with MG after thymectomy, with or without thymoma. By 1954, Keynes had completed and presented more than 200 thymectomies for MG (3).

Keynes was the first to show that MG responds poorly to surgery for thymoma, although the tumour needs to be removed (3). This finding created some confusion and controversy. He described that MG responded better in 
younger patients when a thymoma was present. From then on, MG was routinely treated by thymectomy.

It was believed that many patients showed some improvement 6 to 12 months after surgery. In Europe, MG specialists began to report that MG was remitted in $25 \%$ of patients, improved in another $50 \%$ and unchanged in $25 \%$. The exact mechanism was not completely understood, but it was thought that pathogenetic events in the hyperplastic thymus were interrupted.

\section{Myasthenia gravis (MG): a brief overview of the disease}

MG is an autoimmune disease. It affects the neuromuscular junctions (NMJs) of skeletal muscles, causing muscle weakness and a tendency towards easy fatigability. In most patients $(85 \%)$, autoantibodies directed against acetylcholine receptors (AChRs) are the cause of the disease (4). Patients with AChR-MG experience delay or blockage of normal signalling by way of complement activation, by causing receptor antigenic modulation or by directly blocking receptor function (5).

It has been demonstrated that in $6 \%$ of MG patients, autoantibodies target the muscle-specific kinase (MuSK) protein (MuSK-MG) (6). The rest of MG patients are double-seronegative (dSN-MG), because a detectable level of AChR or MuSK antibodies is absent. In dSN-MG, some patients have low-affinity antibodies against AChR (7).

It has been recently shown that some dSN-MG patients have autoantibodies against low-density lipoprotein receptor-related protein 4 (LRP4), which is a novel autoantigen in MG (8-10). In thymectomystudied populations, measured level of these autoantigens is not seen. Even if these autoantigens are present, due to various factors (differences in antigens, assays used, ethnic origins of the patient and the prescribed medicine prior to thymectomy such as immunosuppressants and corticosteroids), the proportion of LRP4-MG, AChR-MG and MuSK-MG will vary greatly. Clinical data for patient outcomes may be strongly related to the presence of these autoantibodies, and yet the levels present may not be known at the time of the thymectomy. Especially for LRP4-MG, incidence of this novel MG subgroup and thymectomy has rarely been reported (11).

In the NMJ, there is a synapse between the nerve terminal and muscle that is responsible for carrying the nerve signals into muscle contractions. To have the best signal transmission, the AChRs must function properly.
AChRs are responsible for depolarisation in response to nerve stimulation, and they are clustered within the NMJ (12). Clustering of AChRs is mediated by muscle membrane proteins, MuSK proteins and LRP4. For a long time, MuSK has been known to play a key role in all aspects of postsynaptic differentiation. LRP4 is one of the membrane proteins with an effect on neuronal and synaptic development. Specifically, LRP4 is necessary for the development of NMJ (13).

MG is not a homogenous disease. Patients with AChRMG show more thymic pathological findings than patients with MuSK-MG, and differences in the pattern of affected muscles can also be seen in these patients. Furthermore, the response to therapy may vary. For instance, patients with MuSK-MG may demonstrate hypersensitivity when treated with the acetylcholinesterase inhibitor pyridostigmine, whereas they receive great therapeutic benefit from plasma exchange. These MG patients are not good surgical candidates. It is imperative to differentiate the MG subgroups and the unique characteristics of each. This approach may help in both diagnosis and management, especially when thymectomy is the choice of treatment.

\section{Onset and etiology of MG}

Ocular MG is diagnosed in patients with ocular symptoms lasting for at least 2 years. Approximately $15 \%$ of all AChR-MG patients have this type of disease. It has long been proposed that patients with ocular MG are not good candidates for thymectomy. However, several articles have recently proposed that thymectomy is a viable option in the treatment of ocular MG (14). In the generalised form, MG disease can be divided into three groups according to the age of onset, and thymectomy has been the most important treatment modality. In the early-onset form (EOMG), the age of onset is before 50 years. Most patients with EOMG have high levels of anti-AChR antibodies. They also have thymic follicular hyperplasia (15). It is suspected that sex hormones may play a role in this form of the disease, as more than $80 \%$ of patients with follicular hyperplasia are women (16). These patients may also have other autoantibodies and have the potential to develop other autoimmune diseases, such as thyroiditis (17). In the lateonset form (LOMG), the age of onset is more than 50 years. This group more commonly has thymoma. In patients with LOMG, autoantibodies against striated muscle proteins (ryanodine or titin) may be more common (18). The role these proteins play in recurrences of thymoma is not clear. 
LOMG is mostly generalised and severe, with bulbar signs and frequent severe respiratory crises (19). In the verylate-onset form, the age of onset is after 60 years. In the last few decades, MG appearing after age 60 years has been described. Very-late-onset MG predominantly affects males, and it is different than LOMG, because patients do not present with thymoma (20). In a study analysing the 20-year period between 1987 and 2007, it has been demonstrated that the number of patients older than 50 years without thymoma has tripled (21).

During this 20-year period, the incidence of EOMG has not increased in parallel. The increased incidence of verylate-onset MG may be attributed to increased longevity. As autoimmunity increases with age, elderly individuals are more likely to develop an autoimmune disorder such as MG. Although patients with late-onset and very-lateonset MG are routinely thought to be unsuitable candidates for thymectomy, increased longevity may change this perception. These patients may become thymectomy candidates if research studies demonstrate the benefits. Long-term data for these patients are needed.

A single-centre, non-randomised, non-controlled study compared the long-term post-thymectomy results in patients with anti-AChR-positive, anti-AChR-negative and anti-MuSK-positive non-thymomatous MG (22).

Long-term post-thymectomy results were better in patients with anti-AChR antibodies than in those with seronegative disease (22). In seronegative anti-MuSKpositive MG, thymectomy seems to be less effective than in anti-MuSK-negative MG. The role of the thymus in the production of MuSK antibody is not clear, and there is increasing evidence suggesting that thymectomy may not be beneficial in patients with anti-MuSK MG (22).

Different pathogenic mechanisms have been proposed on the basis of the antibody target. Identification of these antibodies created a new classification, which was determined by the type of antibodies. Certainly, these antibodies have implications for the diagnosis, management and prognosis of MG. In EOMG, which is associated with AChR antibodies, it is known that the disease involves the thymus. This may explain why thymectomy is beneficial in the clinical trial reported by Wolfes (23).

With recent developments in patient selection for thymectomy, surgery may be performed in patients who have better potential for positive outcomes. If this occurs, it may be generalised to different subtypes of the disease. Currently, thymectomy is usually recommended for a limited group of patients with AChR antibodies. Remaining patients may benefit from targeted therapies such as rituximab, which has been shown to be effective in the treatment of anti-MuSK-positive patients (24).

It is thought that women have better outcomes after thymectomy. Two articles questioning this belief found no difference in outcome based on gender $(25,26)$. Ironically, the presence of antibody may affect the outcome in patients with anti-MuSK, which is more common in women (27). Some patients with thymoma with MG may not respond very well after surgery, and dismal outcomes may be explained by autoantibodies. Paraneoplasiaassociated antibodies may be present in thymomatous MG, which includes anti-voltage-gated $\mathrm{K}^{+}$and $\mathrm{Ca}^{2+}$ channel, anti-Hu (antineuronal nuclear autoantibody 1), antidihydropyrimidinase-related protein 5 and anti-glutamic acid decarboxylase antibodies (28). Autoantibodies to a voltage-gated $\mathrm{K}^{+}$channel, KCNA4, were demonstrated in Japanese patients with MG with thymoma, along with associated myocarditis and/or myositis (29). In patients with thymoma, even if thymothymectomy completely and permanently removes the tumour tissue, symptoms of MG usually persist and treatment generally requires chronic immunotherapy (4).

\section{What is new in the effects of thymectomy?}

The possible effects of thymectomy and the selection of surgical technique are still debatable issues. In patients with thymic follicular hyperplasia, it has been claimed that thymectomy can be very helpful in changing the course of MG, potentially leading to decreased severity or remission in a significant number of patients (30). It has been consistently reported, and became a well-known reality, that the best outcome may be obtained when the patient has severe thymic hyperplasia and when thymectomy is performed soon after the onset of symptoms $(31,32)$. Thymectomy has the potential to eliminate the main production site of anti-AChR autoantibodies. In this way, thymectomy may result in a decreased level of anti-AChR antibodies, perhaps correlating with the number of B cells available in the thymus of the patient (33). This finding may explain why thymectomy has a better outcome when the patient has severe follicular hyperplasia. However, it has not been shown that the antibody levels are consistently decreased after thymectomy. The long-living plasma cells generated in the thymus that migrate to the periphery may be a reason for this (34).

The possible benefits of thymectomy when compared 
with other medical therapies have been in the centre of the debate, and an international randomised clinical trial to address this debate was strongly encouraged by surgeons and neurologists (35). Enrolment was completed in 2012, and the results were presented in 2016 with a 3 -year followup period. Before the presentation of the paper, it was thought that improvement after thymectomy was related to the elimination of thymic B cells, and it was claimed that other therapies had the potential to produce similar effects. For example, corticosteroids may cause a significant decrease in the size and number of germinal centres in the thymus (36).

Recently, the results of the Thymectomy Trial in NonThymomatous MG Patients Receiving Prednisone Therapy (MGTX) were presented (23). An extended transsternal thymectomy was the selected procedure, because the authors wanted to standardise the procedure for reproducibility and safety. This decision also prevented further discussion about the differences in surgical techniques. The surgery was combined with a standardised prednisone protocol, and the aim of the study was to evaluate if surgery was better than prednisone therapy alone after 3 years. The goal of treatment was to decrease the myasthenic weakness and the dose of prednisone while increasing quality of life.

This study was a randomised, medication-controlled, rater-blinded trial. It demonstrated benefits of thymectomy in MG patients over a period of 3 years. The surgical arm was superior with respect to clinical outcomes, prednisone and azathioprine use, symptoms and distress due to immunosuppressive agents and the need for hospitalisation for exacerbations. It is clear that this study reinforced the essential place of thymectomy in the treatment of MG (23).

\section{Current situation}

The gold standard technique for thymectomy has been described as the transsternal approach. The main advantages of this approach are optimal exposure, complete dissection of the thymus and mediastinal fatty tissue and reproducibility (37). With research claiming zero risk of incomplete thymectomy, a complete recovery from MG may be possible if the cellular (antibody production) and hormonal events occurring in and around the thymus are excluded (37). The risks of major vascular and phrenic nerve injuries should be very low (37). A partial or total sternal splitting technique has several disadvantages, including the splitting of the sternum, longer duration of surgery and longer postoperative hospitalisation. For these reasons, transsternal resections for non-thymomatous thymus have almost been abolished in major thoracic surgery centres and replaced by minimally invasive procedures (23).

Another approach that is rarely used is the transcervical thymectomy. It is popular with younger female patients and neurologists because of some advantages such as short hospitalisation, almost no pain and fewer complications (38). However, the main criticism includes possible incomplete resection of thymus or perithymic fatty tissue because of the crowding of instruments. Although long-term results are comparable with open techniques, most surgeons do not find this technique reproducible.

Video-assisted thoracoscopic surgery (VATS) thymectomy gained popularity after 2000s. It could be performed through a left- or right-sided approach or even subxiphoidal or bilateral approaches. VATS thymectomy has been shown to be an acceptable surgical technique. Metaanalyses and many articles demonstrate that the operation time and intraoperative bleeding is lower in VATS, as well as duration of postoperative ventilation and shorter mean length of stay in hospital when compared with transsternal techniques $(39,40)$. Recently, more support for VATS thymectomy was provided by several authors claiming that extended, or even maximal, thymectomies could be performed with VATS $(41,42)$.

The extent of thymectomy remains a controversial topic. Although the presence of extracapsular thymic tissue is frequent and well described, the accessibility of these ectopic thymic foci is not clear. Also, their function and impact on outcome are not adequately described (43). Ectopic thymic foci are thought to be found more frequently in patients with an atrophic thymus. Their presence may result in a poor outcome, even after maximal thymectomy with resection of all potential sites of ectopic thymic foci in the neck and mediastinum $(44,45)$. Because ectopic thymic foci are usually isolated to one or two sites and are most frequently found in the anterior mediastinal fat, an extended thymectomy involving the fat located between the pericardium, both pleura and the diaphragm can be safely accessible with minimally invasive surgery in the hands of experienced surgeons. It is recommended that these tissues be routinely resected regardless of the type of surgical approach. Extending the surgery to resect tissues in the pericardiophrenic angles, aorto-pulmonary window, aorto-caval groove and along the thyroid gland may cause a risk of phrenic or recurrent nerve injury; thus, some authors claim that there is limited evidence to recommend that it be routinely done (43-45). A randomised clinical trial would 
be needed to answer this question. Interestingly, positron emission tomography (PET) has shown a correlation between parathymic standardised uptake value and the presence of ectopic thymic tissue, but again, future research is needed to determine the clinical utility of PET/computed tomography in patients with MG.

In short, any resection has to include both mediastinal pleuras, a sharp dissection on the pericardium, complete removal of the horns without cutting or coagulating and excision of pericardiophrenic fatty tissues. All thoracic surgeons have to accept the need for a major shift from open surgery to video-assisted surgery and robotic-assisted surgery in non-thymomatous MG. Minimally invasive surgery can be done through a combination of cervicotomy, a transcervical-subxyphoid approach or unilateral or bilateral video-assisted or robotic-assisted thoracic surgery (RATS). The benefits of a minimally invasive approach have clearly demonstrated that it would be unwise to have a randomised trial comparing open and minimally invasive surgery (46-50).

\section{What is our standard approach for thymectomy?}

In our armamentarium, the approaches used for thymectomy for patients with MG are variable. VATS or robotic-assisted thymectomy is preferred, generally from the right side, even when a thymoma is located on the left side. The thymoma can be pulled to the right side after the dissection of the right thymus, or a two-incision exploration can be performed from the left side (51).

The patient is positioned in a $30^{\circ}$ semi-supine position, supported with a roll placed under the right shoulder and the right arm abducted over a support attached to the table if the operation is a VATS thymectomy (51). In this technique, three ports are opened around the mammary gland without violating the mammalian tissue. The first port is always the camera port, and the other ports are opened with the help of a camera. The left port is opened in the anterior axillary fossa, lateral to the pectoralis major muscle. The right port is opened in the 5th-6th intercostal space lateral to the midclavicular line [Figure 1 (51)]. For robotic surgery, the arm is placed inferiorly on the ipsilateral side. Again, three ports are selected without an assistant port.

Our institution has extensive experience with VATS thymectomy. Our knowledge has been replicated and transferred to our experience with robotic surgery. In robotic surgery, an access incision is not opened, using only three ports for a completely robotic thymectomy. With our developing experience, it has been recognised that the left arm of the robot can be placed more anteriorly when compared with the VATS technique. Also, $\mathrm{CO}_{2}$ with a pressure of 7-8 $\mathrm{mmHg}$ is routinely used during robotic surgery but not during VATS. A $30^{\circ}$ up camera angle is used in the beginning, with a $0^{\circ}$ camera angle during the dissection from pericardium and innominate vein, and $30^{\circ}$ down camera angle when dissecting the left phrenic nerve side of the thymus. A single camera at a $30^{\circ}$ angle is used in VATS. A Jackson-Pratt drainage system is used in both techniques after the operation.

Mineo et al. studied patients with left-sided thymectomy and preferred using four flexible trocars (52). Pardolesi et al. preferred RATS with a left-sided approach (53). Three incisions are used at the fifth intercostal space at the anterior axillary line, at the anterior axillary line in the third intercostal space and at the fifth intercostals space at the midclavicular line (53).

\section{Literature findings}

Two studies have compared clinical experience with a rightsided versus a left-sided VATS thymectomy approach in patients with non-thymomatous MG. These studies demonstrated no difference regarding surgical time, intraoperative blood loss, postoperative hospital stay and postoperative complications. Both approaches are feasible, effective and comparable in operative and long-term results for the treatment of non-thymomatous MG (54).

Another study compared a single-port approach from below the xiphoid process and conventional VATS thymectomy. This study demonstrated that operative time was comparable, but the subxiphoid single-port technique had advantages in blood loss, hospitalisation period and need for analgesia in the postoperative period (55). However, there are disadvantages in subxiphoid thymectomy in cases with obesity, cardiomegaly and decreased manoeuvrability of instruments (56).

Toker et al. demonstrated that catastrophic intraoperative complications could occur during VATS thymectomy or thymothymectomy. Although they are rare $(1.5 \%)$, complications are life threatening. The surgical team should be proficient and always ready to convert to open surgery within seconds, managing complications to avoid mortality in these cases (57). Another retrospective study on VATS thymectomy showed that body mass index (BMI) was the sole significant factor related to longer operative time (58). 


\section{Follow-up results after thymectomy}

A meta-analysis compared VATS thymectomy with open thymectomy. Operation time and intensive care unit time were comparable, but intraoperative blood loss and length of hospital stay were lower in VATS. Total number of complications and myasthenic crises were also lower in VATS thymectomy; however, other complications were comparable (39). In a retrospective study of 90 patients who underwent VATS thymectomy, the mean length of chest tube duration and hospitalisation time was $26.7 \pm 18.6 \mathrm{~h}$ and $2.2 \pm 1.1$ days (55). Another study demonstrated that BMI, age, duration of operation and length and duration of drainage are variables related to length of hospital stay in patients who underwent VATS thymectomy (59). As our group increased surgical skill in the VATS thymectomy procedure, we demonstrated that BMI was related to longer operative time and median duration of the operation decreased with increased experience of the surgeon (60).

A study comparing three approaches (RATS-VATS-open surgery) reported that postoperative drainage duration and volume were significantly lower in RATS (2.88 vs. 3.77 and 4.41 days, $\mathrm{P}<0.05 ; 352.2$ vs. 613.9 and $980 \mathrm{~mL}, \mathrm{P}<0.05)$. Hospital stay showed a trend towards shorter duration in RATS compared with the open surgery and VATS (4.3 vs. 5.5 and 6.6 days). However, postoperative complications occurred in three patients in the open surgery group, with no postoperative complications in the RATS and VATS groups (61).

Yu et al. analysed 8 years of follow-up in patients with MG after thymectomy, and they reported that $81.1 \%$ of patients gained satisfactory efficacy, $8.1 \%$ died and $10.8 \%$ remained unchanged or deteriorated. Patients with nonthymomatous MG showed significantly higher rates of complete stable remission and clinical remission than patients with thymomatous MG (62).

Liu et al. also reported that two good prognostic factors were preoperative prescription of anticholinesterase alone $(\mathrm{P}=0.035)$ and non-thymomatous MG $(\mathrm{P}=0.003)$. The 5 -year complete stable remission rates in the VATS group and open group were $56.5 \%$ and $57.1 \%$, respectively (47).

\section{Age and thymectomy}

Thymectomy became a routinely performed procedure for patients with MG. If patients have co-morbidities, surgery can become more complicated. Older age is another factor that complicates the procedure. Moreover, for the patients older than 60 years or with late- or very-late-onset MG, standard thymectomy for MG is not recommended, except in patients with a concomitant thymoma (63). Patients younger than 45 years have a higher rate of achieving complete stable remission during follow-up (64). However, increasing age in the population may soon create a platform for increasing the age limits of thymectomy in patients with MG and without a thymoma.

There seems to be no reported obstacle in younger patients, even in paediatric patients. In Toker et al. the average age was 14.8 (65), and it was 11.3 years in ChristonLagay's study (66). In both studies, all patients underwent thoracoscopic surgery, and this approach was deemed safe in paediatric patients with MG.

\section{New perspectives}

VATS has proved its reliability in thymectomy in MG disease. Usually a three-port approach from the right side is the preferable technique. Single-port thymectomies are also being performed in several minimally invasive surgery centres (67). Scarci et al. described performing a thymectomy from a $3-\mathrm{cm}$ lateral muscle-sparing incision on the 5 th intercostal space using a single-incision laparoscopic surgery port device (67). Another single-port technique is subxiphoid approach uni-portal thymectomy. This technique has advantages for bleeding, duration of operation and requirement of analgesia compared with conventional three-port VATS thymectomy technique (64).

Surgery of the thymic gland in the treatment of MG and thymoma is also entering a new era for robotic-assisted surgery $(68,69)$. It has been shown that robotic thymectomy in patients with MG is safe and feasible. Neurological benefits and decreased need for steroids has been observed by Keijzers (70). A study that compared RATS and VATS thymectomy did not report any statistical difference in conversion to open, length of hospital stays or postoperative pneumonia (71). A meta-analysis on these issues found no significant differences in conversion rates, operation time and length of hospitalisation; however, a slightly higher blood loss was noted in RATS (72).

\section{Conclusions}

VATS has replaced open techniques for surgery of the thymic gland in the treatment of non-thymomatous MG with superiorities for postoperative pain, preserved pulmonary function, stay in hospital and cosmetic results. 
Innovations such as RATS and uni-portal or subxiphoid approaches may produce similar results, if the extent of resection is like VATS with good patient selection and reproducibility. In the future, our understanding of MG may change on the basis of antibodies and new classifications and their relation to the thymus. We are sure this will refine the criteria for better patient selection for thymectomy. The extent of thymectomy will remain an open question. Until another surgical trial in MG demonstrates superiority, basic resection should include both pleura, both horns and mediastinal fatty tissue.

\section{Acknowledgments}

Funding: None.

\section{Footnote}

Provenance and Peer Review: This article was commissioned by the Guest Editor (Tommaso Claudio Mineo) for the series "Mediastinal Surgery" published in fournal of Visualized Surgery. The article has undergone external peer review.

Conflicts of Interest: Both authors have completed the ICMJE uniform disclosure form (available at http://dx.doi. org/10.21037/jovs.2018.07.11). The series "Mediastinal Surgery" was commissioned by the editorial office without any funding or sponsorship. AT serves as an unpaid editorial board member of Gournal of Visualized Surgery from Jun 2017 to May 2019. The authors have no other conflicts of interest to declare.

Ethical Statement: The authors are accountable for all aspects of the work in ensuring that questions related to the accuracy or integrity of any part of the work are appropriately investigated and resolved.

Open Access Statement: This is an Open Access article distributed in accordance with the Creative Commons Attribution-NonCommercial-NoDerivs 4.0 International License (CC BY-NC-ND 4.0), which permits the noncommercial replication and distribution of the article with the strict proviso that no changes or edits are made and the original work is properly cited (including links to both the formal publication through the relevant DOI and the license). See: https://creativecommons.org/licenses/by-nc-nd/4.0/.

\section{References}

1. Schumacher R, Roth J. Thymektomie bei cenem fall von morbus basedowii mit myasthenie. Med Chir 1912;25:746.

2. Kirschner PA. Alfred Blalock and thymectomy for myasthenia gravis. Ann Thorac Surg 1987;43:348-9.

3. Keynes G. Surgery of the thymus gland; second (and third) thoughts. Lancet 1954;266:1197-202.

4. Meriggioli MN, Sanders DB. Autoimmune myasthenia gravis: emerging clinical and biological heterogeneity. Lancet Neurol 2009;8:475-90.

5. Vincent A, Drachman DB. Myasthenia gravis. Adv Neurol 2002;88:159-88.

6. Hoch W, McConville J, Helms S, et al. Autoantibodies to the receptor tyrosine kinase MuSK in patients with myasthenia gravis without acetylcholine receptor antibodies. Nat Med 2001;7:365-8.

7. Leite MI, Jacob S, Viegas S, et al. IgG1 antibodies to acetylcholine receptors in 'seronegative' myasthenia gravis. Brain 2008;131:1940-52.

8. Higuchi O, Hamuro J, Motomura M, et al. Autoantibodies to lowdensity lipoprotein receptor-related protein 4 in myasthenia gravis. Ann Neurol 2011;69:418-22.

9. Zhang B, Tzartos JS, Belimezi M, et al. Autoantibodies to lipoprotein-related protei 4 in patients with doubleseronegative myasthenia gravis. Arch Neurol 2012;69:445-51.

10. Pevzner A, Schoser B, Peters K, et al. Anti-LRP4 autoantibodies in AChR- and MuSK-antibody-negative myasthenia gravis. J Neurol 2012;259:427-35.

11. Zouvelou V, Zisimopoulou P, Rentzos M, et al. Double seronegative myasthenia gravis with anti-LRP 4 antibodies. Neuromuscul Disord 2013;23:568-70.

12. Marques MJ, Conchello JA, Lichtman JW. From plaque to pretzel: fold formation and acetylcholine receptor loss at the developing neuromuscular junction. J Neurosci 2000;20:3663-75.

13. Weatherbee SD, Anderson KV, Niswander LA. LDLreceptor-related protein 4 is crucial for formation of the neuromuscular junction. Development 2006;133:4993-5000.

14. Liu Z, Feng H, Yeung SC, et al. Extended transsternal thymectomy for the treatment of ocular myasthenia gravis. Ann Thorac Surg 2011;92:1993-9.

15. Berrih-Aknin S, Ruhlmann N, Bismuth J, et al. CCL21 overexpressed on lymphatic vessels drives thymic hyperplasia in myasthenia. Ann Neurol 2009;66:521-31.

16. Eymard B, Berrih-Aknin S. Role of the thymus in the 
physiopathology of myasthenia. Rev Neurol (Paris) 1995;151:6-15.

17. Thorlacius S, Aarli JA, Riise T, et al. Associated disorders in myasthenia gravis: autoimmune diseases and their relation to thymectomy. Acta Neurol Scand 1989;80:290-5.

18. Romi F, Skeie GO, Aarli JA, et al. Muscle autoantibodies in subgroups of myasthenia gravis patients. J Neurol 2000;247:369-75.

19. Romi F, Aarli JA, Gilhus NE. Myasthenia gravis patients with ryanodine receptor antibodies have distinctive clinical features. Eur J Neurol 2007;14:617-20.

20. Aragonès JM, Bolíbar I, Bonfill X, et al. Myasthenia gravis: a higher than expected incidence in the elderly. Neurology 2003;60:1024-6.

21. Casetta I, Groppo E, De Gennaro R, et al. Myasthenia gravis: a changing pattern of incidence. J Neurol 2010;257:2015-9.

22. Ponseti JM, Caritg N, Gamez J, et al. A comparison of long-term post-thymectomy outcome of anti-AChRpositive, anti-AChR-negative and anti-MuSK-positive patients with non-thymomatous myasthenia gravis. Expert Opin Biol Ther 2009;9:1-8.

23. Wolfe GI, Kaminski HJ, Sonnett JR, et al. Randomized trial of thymectomy in myasthenia gravis. J Thorac Dis 2016;8:E1782-3.

24. Keung B, Robeson KR, DiCapua DB, et al. Long-term benefit of rituximab in MuSK autoantibody myasthenia gravis patients. J Neurol Neurosurg Psychiatry 2013;84:1407-9.

25. Bachmann K, Burkhardt D, Schreiter I, et al. Thymectomy is more effective than conservative treatment for myasthenia gravis regarding outcome and clinical improvement. Surgery 2009;145:392-8.

26. Luo Y, Pan DJ, Chen FF, et al. Effectiveness of thymectomy in non-thymomatous myasthenia gravis: a systematic review. J Huazhong Univ Sci Technolog Med Sci 2014;34:942-9.

27. Binks S, Vincent A, Palace J. Myasthenia gravis: a clinicalimmunological update. J Neurol 2016;263:826-34.

28. Vernino S, Lennon VA. Autoantibody profiles and neurological correlations of thymoma. Clin Cancer Res 2004;10:7270-5.

29. Suzuki S, Satoh T, Yasouka H, et al. Novel antibodies to a voltage-gated potassium channel Kv1.4 in a severe form ofcmyasthenia gravis. J Neuroimmunol 2005;170:141-9.

30. Spillane J, Hayward M, Hirsch NP, et al. Thymectomy: role in the treatment of myasthenia gravis. J Neurol 2013;260:1798-801.

31. Budde JM, Morris CD, Gal AA, et al. Predictors of outcome in thymectomy for myasthenia gravis. Ann Thorac Surg 2001;72:197-202.

32. Nieto IP, Robledo JP, Pajuelo MC, et al. Prognostic factors for myasthenia gravis treated by thymectomy: review of 61 cases. Ann Thorac Surg 1999;67:1568-71.

33. Okumura $M$, Ohta $M$, Takeuchi $Y$, et al. The immunologic role of thymectomy in the treatment of myasthenia gravis:implication of thymus-associated B-lymphocyte subset in reduction of the anti-acetylcholine receptor antibody titer. J Thorac Cardiovasc Surg 2003;126:1922-8.

34. Newsom-Davis J, Cutter G, Wolfe GI, et al. Status of the thymectomy trial for nonthymomatous myasthenia gravis patients receiving prednisone. Ann N Y Acad Sci 2008;1132:344-7.

35. Fujii Y, Hashimoto J, Monden Y, et al. Specific activation of lymphocytes against acetylcholine receptor in the thymus in myasthenia gravis. J Immunol 1986;136:887-91.

36. Meraouna A, Cizeron-Clairac G, Panse RL, et al. The chemokine CXCL13 is a key molecule in autoimmune myasthenia gravis. Blood 2006;108:432-40.

37. de Perrot M, Donahoe L. Thymectomy for myasthenia gravis: what's next? J Thorac Dis 2017;9:237-9.

38. Masaoka A, Monden Y, Seike Y, et al. Reoperation after transcervical thymectomy for myasthenia gravis. Neurology 1982;32:83-5.

39. Qi K, Wang B, Wang B, et al. Video-assisted thoracoscopic surgery thymectomy versus open thymectomy in patients with myasthenia gravis: a meta-analysis. Acta Chir Belg 2016;116:282-8.

40. Meyer DM, Herbert MA, Sobhani NC, et al. Comparative clinical outcomes of thymectomy for myasthenia gravis performed by extended transsternal and minimally invasive approaches. Ann Thorac Surg 2009;87:385-90; discussion 390-1.

41. Xue L, Pang X, Zhang Y, et al. Extended thymectomy by a cervical incision additional to bilateral VATS approach. J Vis Surg 2017;3:83.

42. Yoshida S, Yoshino I, Moriya Y, et al. Video-Assisted Thoracoscopic Surgery Extended Thymectomy for Myasthenia Gravis Using Manual Manipulators: the Radius Surgical System. Ann Thorac Surg 2011;92:2246-8.

43. de Perrot M, McRae K. Evidence for thymectomy in myasthenia gravis: Getting stronger? J Thorac Cardiovasc Surg 2017;154:314-6. 
44. Tsukada J, Hasegawa I, Sato H, et al. Ectopic cervical thymoma located in the carotid triangle. Jpn J Radiol 2013;31:138-42.

45. Klimek-Piotrowska W, Mizia E, Kuzdzał J, et al. Ectopic thymic tissue in the mediastinum: limitations for the operative treatment of myasthenia gravis. Eur J Cardiothorac Surg 2012;42:61-5.

46. Siwachat S, Tantraworasin A, Lapisatepun W, et al. Comparative clinical outcomes after thymectomy for myasthenia gravis: Thoracoscopic versus trans-sternal approach. Asian J Surg 2018;41:77-85.

47. Liu CW, Luo M, Mei JD, et al. Perioperative and longterm outcome of thymectomy for myasthenia gravis: comparison of surgical approaches and prognostic analysis. Chin Med J (Engl) 2013;126:34-40.

48. Zahid I, Sharif S, Routledge T, et al. Video-assisted thoracoscopic surgery or transsternal thymectomy in the treatment of myasthenia gravis? Interact Cardiovasc Thorac Surg 2011;12:40-6.

49. Lin MW, Chang YL, Huang PM, et al. Thymectomy for non-thymomatous myasthenia gravis: a comparison of surgical methods and analysis of prognostic factors. Eur J Cardiothorac Surg 2010;37:7-12.

50. Orsini B, Santelmo N, Pages PB, et al. Comparative study for surgical management of thymectomy for non-thymomatous myasthenia gravis from the French national database EPITHOR. Eur J Cardiothorac Surg 2016;50:418-22.

51. Kaba E, Cosgun T, Ayalp K, et al. Right sided VATS thymectomy: "current standards of extended thymectomy for myasthenia gravis. Video-assist Thorac Surg 2017;2:30

52. Mineo TC, Pompeo E, Lerut T, et al. Thoracoscopic thymectomy in autoimmune myasthenia: results of leftsided approach. Ann Thorac Surg 2000;69:1537-41.

53. Pardolesi A, Spaggiari L. Left Side Robotic Approach for Extended Thymectomy: Surgical Technique and Preliminary Experience. Austin J Surg 2016;3:1090.

54. Elsayed HH, Gamal M, Raslan S, et al. Video-assisted thoracoscopic thymectomy for non-thymomatous myasthenia gravis: a right-sided or left-sided approach? Interact Cardiovasc Thorac Surg 2017;25:651-3.

55. Suda T, Hachimaru A, Tochii D, et al. Video-assisted thoracoscopic thymectomy versus subxiphoid singleport thymectomy: initial results. Eur J Cardiothorac Surg 2016;49 Suppl 1:i54-8.

56. Ooi A, Sibayan M. Uniportal video assisted thoracoscopic surgery thymectomy (right approach). J Vis Surg
2016;2:13.

57. Özkan B, Toker A. Catastrophes during video-assisted thoracoscopic thymus surgery for myasthenia gravis. Interact Cardiovasc Thorac Surg 2016;23:450-3.

58. Toker A, Tanju S, Sungur Z, et al. Videothoracoscopic thymectomy for nonthymomatous myasthenia gravis: results of 90 patients. Surg Endosc 2008;22:912-6.

59. Toker A, Tanju S, Ziyade S, et al. Early outcomes of videoassisted thoracoscopic resection of thymus in 181 patients with myasthenia gravis: who are the candidates for the next morning discharge? Interact Cardiovasc Thorac Surg 2009;9:995-8.

60. Toker A, Tanju S, Ziyade S, et al. Learning curve in videothoracoscopic thymectomy: how many operations and in which situations? Eur J Cardiothorac Surg 2008;34:155-8.

61. Qian L, Chen X, Huang J et al. A comparison of three approaches for the treatment of early-stage thymomas: robot-assisted thoracic surgery, video-assisted thoracic surgery, and median sternotomy. J Thorac Dis 2017;9:1997-2005.

62. Yu S, Li F, Chen B, et al. Eight-year follow-up of patients with myasthenia gravis after thymectomy. Acta Neurol Scand 2015;131:94-101.

63. Alkhawajah NM, Oger J. Treatment of Myasthenia Gravis in the Aged. Drugs Aging 2015;32:689-97.

64. Diaz A, Black E, Dunning J. Is thymectomy in nonthymomatous myasthenia gravis of any benefit? Interact Cardiovasc Thorac Surg 2014;18:381-9.

65. Özkan B, Demir A, Kapdagli M, et al. Results of videothoracoscopic thymectomy in children: an analysis of 40 patients. Interact Cardiovasc Thorac Surg 2015;21:292-5.

66. Christison-Lagay E, Dharia B, Vajsar J, Kim PC. Efficacy and safety of thoracoscopic thymectomy in the treatment of juvenile myasthenia gravis. Pediatr Surg Int 2013;29:583-6.

67. Bedetti B, Solli P, Lawrence D, et al. Single port videoassisted thoracoscopic thymectomy. J Vis Surg 2016;2:149.

68. Kaba E, Cosgun T, Ayalp K, et al. Robotic thymectomy-a new approach for thymus. J Vis Surg 2017;3:67.

69. Ismail M, Swierzy M, Rückert RI, et al. Robotic thymectomy for myasthenia gravis. Thorac Surg Clin 2014;24:189-95.

70. Keijzers M, de Baets M, Hochstenbag M, et al. Robotic thymectomy in patients with myasthenia gravis: neurological and surgical outcomes. Eur J Cardiothorac 
Surg 2015;48:40-5.

71. Fok M, Bashir M, Harky A, et al. Video-Assisted Thoracoscopic Versus Robotic-Assisted Thoracoscopic Thymectomy: Systematic Review and Meta-analysis.

doi: 10.21037/jovs.2018.07.11

Cite this article as: Cosgun T, Toker A. Video-assisted thoracoscopic thymectomy for myasthenia gravis: where we are. J Vis Surg 2018;4:165.
Innovations (Phila) 2017;12:259-264.

72. Buentzel J, Heinz J, Hinterthaner M, et al. Robotic versus thoracoscopic thymectomy: The current evidence. Int J Med Robot 2017;13(4). 